\title{
Analytical presentation of resistive impedance for the laminated vacuum chamber
}

\author{
Mikayel Ivanyan \\ Center for the Advancement of Natural Discoveries using Light Emission, CANDLE, 375040, Yerevan, Armenia
}

Andranik Tsakanian

Yerevan State University, 375025, Yerevan, Armenia

(Received 12 August 2005; published 22 March 2006)

\begin{abstract}
The analytical expressions for the multipole longitudinal and transverse impedances of two-layer laminated vacuum chamber are obtained in ultrarelativistic limit. A number of special cases are derived that coincide with the well-known solutions. A numerical example for the impedance of stainless steelcopper laminated vacuum chamber is given.
\end{abstract}

DOI: 10.1103/PhysRevSTAB.9.034404

PACS numbers: 41.60.- m, 41.75.Ht, 42.82.Et

\section{INTRODUCTION}

The stable operation of advance accelerator facilities requires careful examination of vacuum chamber longitudinal and transverse impedances that can drive various types of beam instabilities [1,2]. Very often the technical solutions (high vacuum performance, cure of static charge, reduction of the impedance, etc.) imply the use of laminated vacuum chamber.

The analytical solutions for impedance are known for a number of structures: two parallel resistive plates [3], circular [4], and elliptic [5] single-layer resistive cylinders. The longitudinal impedance for two-layer tube with perfectly conducting outer layer is given in Ref. [6]. In Ref. [7] the transverse dipole impedance for two-layer pipe with infinite outer layer thickness is evaluated analytically for a number of special cases. Recently the exact analytical solution for the monopole term of longitudinal impedance of two-layer pipe is obtained independently in Refs. [8,9] for the relativistic charge. In [8] the transformation of the solution to ultrarelativistic case is performed.

In this paper, the analytical solution for the multipole impedance (longitudinal and transverse) of two-layer resistive tube with walls finite thickness is derived. A number of special cases in ultrarelativistic limit are evaluated analytically that coincide with well-known solutions. A numerical example for impedance of stainless-steel-copper two-layer vacuum chamber is given.

The common approach to numerically evaluate the impedance of vacuum chamber with laminated walls is the field matching technique. The corresponding general numerical algorithm for a straight, uniform beam in a concentric, circular-cylindrical multilayer tube is given in $[2,10,11]$. However the numerical application of this method is valid for the relativistic charge only (Lorenz factor $\gamma$ is finite), as the fields in vacuum outside the tube are diverge in ultrarelativistic limit $(\gamma=\infty)$. In this paper, the transformation to ultrarelativistic limit is performed after derivation of the analytical solution for the impedance.
In our calculations the classical macroscopic electrodynamics is used valid for the frequency range of $\omega \ll$ $c / a_{0}$, where $c$ is the velocity of light, $a_{0}$ is the distance between atoms of layers material. In addition, an ultrarelativistic limit $\gamma=\infty$ assumes the charge energy infinity, resulting in impedance that reaches to infinite frequencies. As in reality the charge has finite (large) $\gamma$, the impedance in ultrarelativistic limit will be cut off at frequencies $\omega \sim$ $\gamma c / b$ with $b$ - the vacuum chamber radius.

\section{IMPEDANCES AND SYNCHRONOUS COMPONENTS}

Consider the relativistic plane disk of radius $a_{1}$, total charge $Q$, and the charge transverse distribution $\rho(r, \theta)$ moving with velocity $v(v \sim c)$ along the $z$ axis of uniform, circular-cylindrical two-layer tube of inner radius $a_{2}=b$ (Fig. 1). The disk center coincides with the tube axis and $(r, \theta, z)$ is the cylindrical coordinates. The charge distribution is then given by $Q(r, \theta, z)=Q \rho(r, \theta) \delta(z-$ $v t$ ), where the transverse distribution $\rho(r, \theta)$ is normalized to unity. The boundary between two layers is located at $r=$
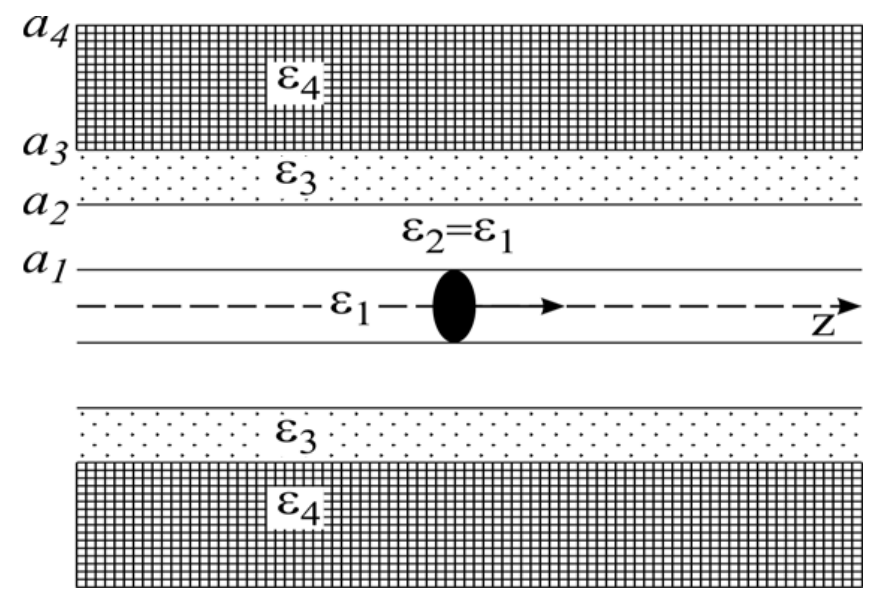

FIG. 1. Geometry of the problem. 
$a_{3}$ and the outer radius of the tube is $a_{4}$. The layers can be either dielectrics or metals. Outside of the tube is vacuum.

The cross section of the tube is divided into five concentric regions: (1) $0 \leq r \leq a_{1}=r_{s}$ (vacuum), (2) $a_{1} \leq$ $r \leq a_{2}=b$ (vacuum), (3) $a_{2} \leq r \leq a_{3}$ (first layer), (4) $a_{3} \leq r \leq a_{4}$ (second layer), and (5) $r \geq a_{4}$ (vacuum).

In general, due to current axial asymmetry the fields radiated in the tube have all six components $E_{z}, H_{z}$, $E_{\varphi}, H_{\varphi}$, and $E_{r}, H_{r}$.

The longitudinal $Z_{\|}(\omega, \vec{r})$ and transverse $\vec{Z}_{\perp}(\omega, \vec{r})$ impedances of the structure are defined as the Fourier transformation of longitudinal $W_{\|}(s, \vec{r})$ and transverse wake $\vec{W}_{\perp}(s, \vec{r})$ potentials produced by the exciting charge and are given by

$$
\begin{aligned}
& Z_{\|}(\omega, \vec{r})=\frac{1}{v} \int_{-\infty}^{\infty} W_{\|}(\vec{r}, s) e^{-j \omega s / v} d s, \\
& \vec{Z}_{\perp}(\omega, \vec{r})=-\frac{j}{v} \int_{-\infty}^{\infty} \vec{W}_{\perp}(\vec{r}, s) e^{-j \omega s / v} d s,
\end{aligned}
$$

where integration over time variable $t$ is replaced by the distance $s=v t-z$ and the factor $(-j)$ for transverse impedance is used for convenience.

Taking into account that the wake potentials are given by the integrated Lorenz force acting on the test charge moving at the distance $s$ behind the exciting charge as

$$
\begin{aligned}
W_{\|}(s, r) & =-\frac{1}{Q} \int_{-\infty}^{\infty} E_{z}(r, z, t)_{t=(z+s) / v} d z, \\
\vec{W}_{\perp}(s, r) & =\frac{1}{Q} \int_{-\infty}^{\infty}\left[\vec{E}+\frac{1}{c}(\vec{v} \times \vec{H})\right]_{\perp t=(z+s) / v} d z
\end{aligned}
$$

the impedances can be expressed by the Fourier components of the excited electromagnetic fields

$$
\begin{aligned}
& Z_{\|}(\omega, \vec{r})=-\frac{1}{Q} \int_{-\infty}^{\infty} E_{z \omega}(\vec{r}, z) e^{j \omega z / v} d z, \\
& \vec{Z}_{\perp}(\omega, \vec{r})=\frac{-j}{Q} \int_{-\infty}^{\infty}\left[\vec{E}_{\omega}+\frac{1}{c}\left(\vec{v} \times \vec{H}_{\omega}\right)\right]_{\perp} e^{j \omega z / v} d z .
\end{aligned}
$$

The Panofsky-Wenzel theorem gives the relation between the longitudinal and transverse impedances as [12]

$$
\vec{Z}_{\perp}(\omega, \vec{r})=\frac{v}{\omega} \nabla_{\perp} Z_{\|}(\omega, \vec{r}) .
$$

In beam existing region $(i=1)$ the frequency domain longitudinal electric field satisfies inhomogeneous wave equation

$$
\Delta E_{z \omega}+\frac{\omega}{c^{2}} E_{z \omega}=\frac{j Q \lambda^{2}}{\varepsilon_{0} \omega} \rho(r, \theta) \exp (-j \omega z / v),
$$

with $\varepsilon_{0}$-the vacuum dielectric constant, $k=\omega / v, \lambda=$ $k / \gamma$ and $\gamma$-the Lorenz factor. In a longitudinally uniform pipe the dependence of electromagnetic fields on coordinate $z$ is given by $\exp (-i p z)$ with $p$-the propagation constant. The general solution of homogeneous wave equation finite at the structure axis $r=0$, are given by the combination of the first kind modified Bessel functions $I_{m}(\xi r)$ and multipolar azimuthal components $\cos m \theta$

$$
E_{z \omega m}^{(h)}(r, \theta, z)=A I_{m}(\xi r) \cos m \theta \exp (-j p z),
$$

with $\xi^{2}=p^{2}-\omega^{2} / c^{2}$ the transverse wave number. As seen from (3) only the synchronous component of the fields $(p=\omega / v)$ contribute into the impedance and the transverse wave number for synchronous fields is simply $\xi=\lambda$.

We assume that the $m$ th mode of synchronous electromagnetic field in region $r \leq a_{1}$ is excited by the $m$-pole moment of charge transverse distribution

$$
\rho_{m}(r, \theta)=\frac{q_{m}}{T_{m}} r^{m} \cos m \theta,
$$

where

$$
\begin{aligned}
q_{m} & =\int_{0}^{2 \pi} \int_{0}^{a_{1}} \rho(r, \theta) r^{m+1} \cos m \theta d r d \theta, \\
T_{m} & =\frac{\pi a_{1}^{2(m+1)}}{2(m+1)} .
\end{aligned}
$$

The $m$-mode partial solution is then given by

$$
E_{z \omega m}^{(p)}(r, \theta, z)=\frac{j Q q_{m}}{\varepsilon_{0} \omega T_{m}} r^{m} \cos m \theta \exp (-j \omega z / v) .
$$

For the particular case of point charge $Q$ moving in parallel with $z$ axis and the offset $a_{1}, q_{m}=\delta_{m} a_{1}^{m}$ with $\delta_{0}=1 / 2$ and $\delta_{m>0}=1$.

\section{FIELDS IN TWO-LAYER VACUUM CHAMBER}

Following the results of the previous section and Maxwell equations, the frequency domain multipole components of the electromagnetic fields, synchronously moving with the charge can be represented as

$$
\begin{aligned}
\left\{E_{r}, E_{z}, H_{\theta}\right\}_{m}(\omega, r, \theta, z)= & \left\{E_{r}, E_{z}, H_{\theta}\right\}_{m}(\omega, r) \\
& \times \cos m \theta \exp (-j \omega z / v), \\
\left\{H_{r}, H_{z}, E_{\theta}\right\}_{m}(\omega, r, \theta, z)= & \left\{H_{r}, H_{z}, E_{\theta}\right\}_{m}(\omega, r) \\
& \times \sin m \theta \exp (-j \omega z / v) .
\end{aligned}
$$

The $m$-pole component of longitudinal impedance per unit structure length $\tilde{Z}_{\|}$is then expressed as

$$
\tilde{Z}_{\| m}(\omega, r, \theta)=-\frac{1}{Q} E_{z m}(\omega, r) \cos m \theta .
$$

To obtain the structure impedances we therefore need to define the radial dependence of the frequency domain $m$-pole longitudinal electric field.

Omitting the factors $\exp (-j \omega z / v), \cos m \theta$ and $\sin m \theta$ from further considerations, the radial dependence of the frequency domain $m$-pole longitudinal electrical and magnetic components $E_{z m}^{(i)}, H_{z m}^{(i)}$ in each region $(i=1,2,3,4,5)$ are given by equations 


$$
\begin{aligned}
& {\left[\frac{1}{r} \frac{\partial}{\partial r}\left(r \frac{\partial}{\partial r}\right)-\frac{m^{2}}{r^{2}}-\chi_{i}^{2}\right] E_{z m}^{(i)}=\frac{j Q \chi_{i}^{2}}{\omega \varepsilon_{i} T_{m}} q_{m}^{(i)} r^{m},} \\
& {\left[\frac{1}{r} \frac{\partial}{\partial r}\left(r \frac{\partial}{\partial r}\right)-\frac{m^{2}}{r^{2}}-\chi_{i}^{2}\right] H_{z m}^{(i)}=0,}
\end{aligned}
$$

where $q_{m}^{(1)}=q_{m}, q_{m}^{(i)}=0$ for $i>1, \varepsilon_{i}$ are dielectric permeability, $\chi_{i}=\sqrt{k^{2}-\mu_{0} \varepsilon_{i} \omega^{2}}\left(\operatorname{Re}\left(\chi_{i}\right)>0\right)$ are radial propagation constants, $\mu_{0}$ is the magnetic permeability of vacuum. In vacuum regions $(i=1,2,5): \varepsilon_{i}=\varepsilon_{0}$ and $\chi_{i}=k / \gamma=\lambda$. The radial dependence of the transverse components of electric and magnetic fields is then expressed as

$$
\begin{aligned}
& E_{r m}=j k \chi_{i}^{-2}\left(\frac{\partial E_{z m}}{\partial r}+v \mu_{0} \frac{m}{r} H_{z m}\right), \\
& E_{\theta m}=-j k \chi_{i}^{-2}\left(v \mu_{0} \frac{\partial H_{z m}}{\partial r}+\frac{m}{r} E_{z m}\right), \\
& H_{r m}=j k \chi_{i}^{-2}\left(\frac{\partial H_{z m}}{\partial r}+v \varepsilon_{i} \frac{m}{r} E_{z m}\right), \\
& H_{\theta m}=j k \chi_{i}^{-2}\left(v \varepsilon_{i} \frac{\partial E_{z m}}{\partial r}+\frac{m}{r} H_{z m}\right) .
\end{aligned}
$$

The right-hand side of Eq. (12) for the electrical component vanishes everywhere except in the beam region with nonzero charge density $\rho(r, \theta)$. Therefore the nonzero partial solution exists only in the beam region that is simply $E_{z m}=G_{1}(r)=j Q q_{m} r^{m} /\left(\omega \varepsilon_{0} T_{m}\right)$. The solution of the homogeneous wave equation in the beam region, which includes the axis $r=0$, is only modified Bessel functions of first kind since those of the second kind diverge for argument zero. The longitudinal electric and magnetic fields in the beam region are then

$$
E_{z m}^{(1)}(r)=F_{1} I_{m}(\lambda r)+G_{1}(r), \quad H_{z m}^{(1)}(r)=P_{1} I_{m}(\lambda r)
$$

with $F_{1}, P_{1}$ unknown coefficients.

In the subsequent regions $2,3,4$, the longitudinal field components are given by superposition of modified Bessel functions of both kinds:

$$
\begin{aligned}
& E_{z m}^{(i)}=\left[F_{i} R_{i}(r)+G_{i} S_{i}(r)\right], \\
& H_{z m}^{(i)}=\left[P_{i} R_{i}(r)+Q_{i} S_{i}(r)\right], \quad i=2,3,4
\end{aligned}
$$

with $F_{i}, G_{i}, P_{i}, Q_{i}$ unknown coefficients and the functions $R_{i}(r), S_{i}(r)$ combined as

$$
\begin{aligned}
& R_{i}(r)=K_{m}\left(\chi_{i} a_{i}\right) I_{m}\left(\chi_{i} r\right)-I_{m}\left(\chi_{i} a_{i}\right) K_{m}\left(\chi_{i} r\right), \\
& S_{i}(r)=K_{m}^{\prime}\left(\chi_{i} a_{i}\right) I_{m}\left(\chi_{i} r\right)-I_{m}^{\prime}\left(\chi_{i} a_{i}\right) K_{m}\left(\chi_{i} r\right) .
\end{aligned}
$$

Transverse components (13) can be expressed via the following combinations

$$
\begin{aligned}
& R_{i}^{\prime}(r)=K_{m}\left(\chi_{i} a_{i}\right) I_{m}^{\prime}\left(\chi_{i} r\right)-I_{m}\left(\chi_{i} a_{i}\right) K_{m}^{\prime}\left(\chi_{i} r\right), \\
& S_{i}^{\prime}(r)=K_{m}^{\prime}\left(\chi_{i} a_{i}\right) I_{m}^{\prime}\left(\chi_{i} r\right)-I_{m}^{\prime}\left(\chi_{i} a_{i}\right) K_{m}^{\prime}\left(\chi_{i} r\right) .
\end{aligned}
$$

In the outer region $(i=5)$ that extends to infinity, only modified Bessel functions of the second kind are admissible. The longitudinal fields in outer region are then

$$
E_{z m}^{(5)}=F_{5} K_{m}(\lambda r), \quad H_{z m}^{(5)}=P_{5} K_{m}(\lambda r) .
$$

From five field components $E_{z}, H_{z}, E_{\varphi}, H_{\varphi}$, and $H_{r}$ which should be matched at transition boundaries, the four ones have been chosen for basic equations system composition, providing matching at $r=a_{i}(i=1,2,3,4)$ :

$$
\begin{cases}E_{z m}^{(i)}=E_{z m}^{(i+1)}, & H_{z m}^{(i)}=H_{z m}^{(i+1)}, \\ H_{\theta m}^{(i)}=H_{\theta m}^{(i+1)}, & H_{r m}^{(i)}=H_{r m}^{(i+1)} .\end{cases}
$$

The matching of $E_{\theta m}$ component follows automatically. The matching conditions (19) in terms of $m$-pole components of longitudinal electric $E_{z m}$ and magnetic $H_{z m}$ fields are read as

$$
\begin{aligned}
E_{z m}^{(i)}\left(a_{i}\right)= & E_{z m}^{(i+1)}\left(a_{i}\right), \quad H_{z m}^{(i)}\left(a_{i}\right)=H_{z m}^{(i+1)}\left(a_{i}\right), \\
& \frac{1}{\chi_{i}^{2}}\left[\frac{m}{a_{i}} H_{z m}^{(i)}\left(a_{i}\right)+v \varepsilon_{i} \frac{\partial E_{z}^{(i)}\left(a_{i}\right)}{\partial r}\right] \\
= & \frac{1}{\chi_{i+1}^{2}}\left[\frac{m}{a_{i}} H_{z m}^{(i+1)}\left(a_{i}\right)+v \varepsilon_{i+1} \frac{\partial E_{z m}^{(i+1)}\left(a_{i}\right)}{\partial r}\right], \\
& \frac{1}{\chi_{i}^{2}}\left[\frac{\partial H_{z m}^{(i)}\left(a_{i}\right)}{\partial r}+\frac{m}{a_{i}} v \varepsilon_{i} E_{z m}^{(i)}\left(a_{i}\right)\right] \\
= & \frac{1}{\chi_{i+1}^{2}}\left[\frac{\partial H_{z m}^{(i+1)}\left(a_{i}\right)}{\partial r}+\frac{m}{a_{i}} v \varepsilon_{i+1} E_{z m}^{(i+1)}\left(a_{i}\right)\right]
\end{aligned}
$$

for boundaries $i=1, \ldots, 4$. The result is the system of 16 linear algebraic equations with 16 unknown coefficients $F_{i}, P_{i}(i=1, \ldots, 5)$, and $Q_{i}, G_{i}(i=2,3,4)$. The common solution of this system has a complex form and is solved sequentially. The modification of the system to find the unknown coefficients is given in the appendix. In particular for the coefficient $F_{1}$ we obtain

$$
F_{1}=-G_{1}\left(a_{1}\right) \frac{A_{m}(\lambda, k)}{I_{m}\left(\lambda a_{2}\right)},
$$

where the function $A_{m}(\lambda, k)$ is a complicated combination of Bessel functions and in general can be evaluated analytically. The $m$-pole component of longitudinal electric field is then given by

$$
E_{z m}^{(1)}(r, k)=G_{1}(r)-G_{1}\left(a_{1}\right) \frac{I_{m}(\lambda r)}{I_{m}\left(\lambda a_{2}\right)} A_{m}(\lambda, k),
$$

where

$$
G_{1}(r)=j Q \frac{q_{m} r^{m}}{\varepsilon_{0} v k T_{m}}=j Q \frac{q_{m}}{\varepsilon_{0} v k}\left(\frac{r}{a_{1}}\right)^{m} \frac{2(m+1)}{\pi a_{1}^{m+2}} .
$$

An important feature of the function $A_{m}(\lambda, k)$ is its finite 
value in ultrarelativistic limit $\lambda \rightarrow 0$ :

$$
A_{m}(\lambda, k) \underset{\lambda \rightarrow 0}{\rightarrow}\left(\frac{a_{2}}{a_{1}}\right)^{m}\left[1+\frac{1}{m+1} \frac{a_{1}^{2}}{U_{m}(k)}\left(\frac{a_{1}}{a_{2}}\right)^{2 m}\right]
$$

The longitudinal electric field in ultrarelativistic limit is then given by

$$
E_{z m}^{(1)}(r, k)=-\frac{j Q}{\pi \varepsilon_{0} c k U_{m}(k)} \frac{q_{m}}{a_{1}^{m}}\left(\frac{r}{a_{2}}\right)^{m}\left(\frac{a_{1}}{a_{2}}\right)^{m} .
$$

Note, that the transition to the limiting form of $U_{m}$ for monopole $(m=0)$ and multipole $(m>0)$ terms should be performed separately. The function $U_{m}(k)$ is given explicitly in the following section.

\section{IMPEDANCES}

The $m$-pole component of longitudinal impedance per unit length is then derived as

$$
\tilde{Z}_{z m}(r, k)=\frac{1}{Q} E_{z m}^{(1)}(r, k)=\left(\frac{r}{a_{2}}\right)^{m}\left(\frac{a_{1}}{a_{2}}\right)^{m} \frac{q_{m}}{a_{1}^{m}} Z_{z m}(k)
$$

with the frequency dependent part given by

$$
Z_{z m}(k)=-\frac{j Z_{0}}{\pi k U_{m}(k)},
$$

where $Z_{0}=377 \Omega$ is an impedance of free space.

The transverse modes are given by Panofsky-Wenzel theorem [12]

$$
\tilde{Z}_{\perp, m}(k, r, \theta)=k^{-1} \nabla_{\perp}\left[\tilde{Z}_{z m}(k, r) \cos m \theta\right]
$$

that is read as

$$
\begin{aligned}
\tilde{\bar{Z}}_{\perp m}(k, r, \theta)= & \frac{q_{m}}{a_{1}^{m}} \frac{m}{k a_{2}} Z_{z m}(k)\left(\frac{a_{1}}{a_{2}}\right)^{m}\left(\frac{r}{a_{2}}\right)^{m-1} \\
& \times\left[\cos m \theta \vec{i}_{r}-\sin m \theta \vec{i}_{\theta}\right],
\end{aligned}
$$

with $\vec{i}_{r}, \vec{i}_{\theta}$ unit vectors in $r$ and $\theta$ directions, respectively.

The frequency dependent part of transverse impedance is then simply derived as

$$
Z_{\perp m}(k)=\frac{m}{k a_{2}} Z_{z m}(k) .
$$

\section{A. Longitudinal monopole}

In case of $m=0$ the ultrarelativistic limit for the monopole term is obtained in [8]

$$
\begin{aligned}
U_{0}= & a_{2}^{2}-2 \frac{a_{2} \varepsilon_{3}}{\chi_{3} \varepsilon_{0}} \\
& \times \frac{\varepsilon_{3} \chi_{4} R_{4}\left(a_{3}\right) S_{3}^{\prime}\left(a_{2}\right)-\varepsilon_{4} \chi_{3} R_{3}^{\prime}\left(a_{2}\right) R_{4}^{\prime}\left(a_{3}\right)}{\varepsilon_{3} \chi_{4} R_{4}\left(a_{3}\right) S_{3}\left(a_{2}\right)-\varepsilon_{4} \chi_{3} R_{3}\left(a_{2}\right) R_{4}^{\prime}\left(a_{3}\right)} .
\end{aligned}
$$

For metallic layers with skin depth much smaller than the tube radii the impedance is modified to

$$
U_{0}=a_{2}^{2}+2 \frac{a \varepsilon_{3}}{\varepsilon_{0} \chi_{3}} \frac{1+\alpha_{0} t h \chi_{3} d_{3} t h \chi_{4} d_{4}}{t h \chi_{3} d_{3}+\alpha_{0} t h \chi_{4} d_{4}}
$$

where $\quad d_{3}=a_{3}-a_{2}, \quad d_{4}=a_{4}-a_{3} \quad$ and $\alpha_{0}=$ $\left(\chi_{4} \varepsilon_{3}\right) /\left(\chi_{3} \varepsilon_{4}\right) \approx\left(\sigma_{3} / \sigma_{4}\right)^{1 / 2}$ with $\sigma_{3}$ and $\sigma_{4}$ the layers metal conductivities.

\section{B. Longitudinal multipole}

In case of $m>1$ the ultrarelativistic presentation of function $U_{m}(k)$ is given by

$$
U_{m}=\frac{a_{2}^{2}}{m+1}+\frac{m}{\chi_{3}^{2}} \frac{\varepsilon_{03}}{\varepsilon_{0}}-\frac{a_{2}}{\varepsilon_{0} \chi_{3}}\left(\varepsilon_{03} \frac{R_{3}^{\prime}}{R_{3}}-\frac{g^{2}}{a_{2} \chi_{3}} \frac{\Omega}{\varepsilon_{4} R_{3}^{2} M}\right)
$$

and

$$
\begin{aligned}
& \Omega=(m-1) \varepsilon_{0} \varepsilon_{34}^{2}+\varepsilon_{04} g_{m}\left[m \varepsilon_{3} \varepsilon_{4} A R_{4}+a_{3} \chi_{4}\left(\varepsilon_{3}^{2} T_{3}\right.\right. \\
& \left.\left.+\varepsilon_{0} \varepsilon_{4} T_{2}\right)\right] \text {, } \\
& M=a_{3}^{2} \chi_{4}^{2}\left\{\left[\varepsilon_{04} m(m-1)+\varepsilon_{0} a_{4}^{2} \chi_{4}^{2}\right] T_{2} T_{3}+\varepsilon_{04} a_{4} \chi_{4}(m\right. \\
& \left.-1)\left(T_{3} S_{4}^{\prime}-\alpha_{0} p T_{4} R_{4}\right)\right\}+(m-1)\left(\varepsilon_{03} a_{3} \chi_{4} g p\right. \\
& \left.-m \varepsilon_{4} A\right)-m^{2} \varepsilon_{04} g_{m} B R_{4}, \\
& g_{m}=a_{4} \chi_{4}(m-1) S_{4}+\left[m(m-1)+\frac{\varepsilon_{0}}{\varepsilon_{04}} a_{4}^{2} \chi_{4}^{2}\right] R_{4}, \\
& T_{2}=R_{4}^{\prime}-\alpha_{0} p R_{4}, \quad T_{3}=R_{4}^{\prime}-g p R_{4} . \\
& T_{4}=S_{4}^{\prime}-g p S_{4}, \quad A=\left(1-\alpha_{0} g^{2}\right)+\frac{\varepsilon_{0}}{\varepsilon_{4}}\left(1-g^{2}\right), \\
& B=\left(1-\alpha_{0} g\right)\left(1-g^{2}\right),
\end{aligned}
$$

\section{SPECIAL CASES}

In this section, some special cases are evaluated that directly follow from the presentation of the multipole impedance.

\section{A. Two-layer tube: Dipole mode}

For the dipole mode ( $m=1)$ the function (33) is essentially simplified to

$$
\begin{aligned}
U_{1}= & a_{2}^{2}+\frac{\varepsilon_{03}}{\varepsilon_{0} \chi_{3}^{2}}\left(1-\chi_{3} a_{2} \frac{R_{3}^{\prime}}{R_{3}}-\frac{g^{2}}{a_{2} \chi_{3}} \frac{R_{4}}{R_{3}}\right. \\
& \left.\times \frac{\varepsilon_{3} \varepsilon_{4} A R_{4}+a_{3} \chi_{4}\left(\varepsilon_{3}^{2} T_{3}+\varepsilon_{0} \varepsilon_{4} T_{2}\right)}{\varepsilon_{03} \varepsilon_{4} R_{3}\left(a_{3}^{2} \chi_{4}^{2} T_{2} T_{3}-R_{4}^{2} B\right)}\right) .
\end{aligned}
$$

For the metallic type layers, the dielectric permeability $\varepsilon_{i}$ and the radial propagation constants $\chi_{i}(i=3,4)$ are given by $\varepsilon_{i}=\varepsilon_{0}-j \sigma_{i} / \omega, \chi_{i}=\sqrt{j \mu_{0} \bar{\sigma}_{i} \omega}$ where $\sigma_{i}$ are layers' conductivities, $\mu_{0}$ is the vacuum magnetic permeability 
and the positive real part of squared root is assumed. A good approximation for metallic type two-layer tube is the smallness of each layer skin depth $\delta_{i}=\left(2 / \sigma \mu_{0} \omega\right)^{1 / 2}$ with respect to the layer inner radius $a_{i}$, i.e., $\delta_{i} / a_{i} \ll 1$. Denoting $t_{1}=\operatorname{th}\left(d_{3} \chi_{3}\right), t_{2}=\operatorname{th}\left(d_{4} \chi_{4}\right)$ the expression (34) for the dipole mode is modified to

$$
\begin{aligned}
U_{1}= & \frac{a_{2}^{2}}{2}+\frac{1}{\chi_{3}^{2}} \frac{\varepsilon_{03}}{\varepsilon_{0}}-\frac{a_{2}}{\varepsilon_{0} \chi_{3}} \\
& \times \frac{\left(B \varepsilon_{03} t_{1}-g A \varepsilon_{3} a_{3} \chi_{4}\right) t_{2}^{2}-a_{3}^{2} \chi_{4}^{2}\left(\varepsilon_{0} u_{1} u_{2}+\varepsilon_{3} u_{3} u_{4}\right)}{a_{3}^{2} \chi_{4}^{2} u_{1} u_{3}-B t_{1}^{2} t_{2}^{2}}
\end{aligned}
$$

with $u_{1}=t_{1}+\alpha_{0} t_{2}, u_{2}=1+g t_{1} t_{2}, u_{3}=t_{1}+g t_{2}, u_{4}=$ $1+\alpha_{0} t_{1} t_{2}$. By substituting $t_{2}=1$ in (35), the impedance of two-layer tube with infinite outer layer thickness is derived.

\section{B. Single-layer tube}

For the single-layer tube, the impedance of multipole modes is obtained by substituting in (33) $\varepsilon_{2}=\varepsilon_{3}$ and $\chi_{2}=\chi_{3}$ or $a_{4}=a_{3}$. The result for the single-layer tube with wall propagation constant $\chi$ and dielectric constant $\varepsilon$ is read as

$$
\begin{aligned}
U_{m}= & \frac{a_{2}^{2}}{m+1}+\frac{1}{\varepsilon_{0} \chi^{2}}\left(m\left(\varepsilon_{0}+\varepsilon\right)\right. \\
& \left.+\frac{4 \varepsilon_{0} \varepsilon(m-1)-a_{2} \chi\left(\varepsilon_{0}+\varepsilon\right)^{2}\left(c_{1} R^{\prime}+c_{2} S^{\prime}\right) R}{\left(\varepsilon_{0}+\varepsilon\right)\left(c_{1} R+c_{2} S\right) R}\right),
\end{aligned}
$$

with $\left\{R, R^{\prime}, S, S^{\prime}\right\}=\left\{R_{3}\left(a_{2}\right), R_{3}^{\prime}\left(a_{2}\right), S_{3}\left(a_{2}\right), S_{3}^{\prime}\left(a_{2}\right)\right\}$ (see Eqs. (16) and (17)

$$
c_{1}=m(m-1)+\frac{\varepsilon_{0}}{\varepsilon_{0}+\varepsilon} a_{3}^{2} \chi^{2}, c_{2}=(m-1) a_{3} \chi .
$$

The dipole mode is then given by

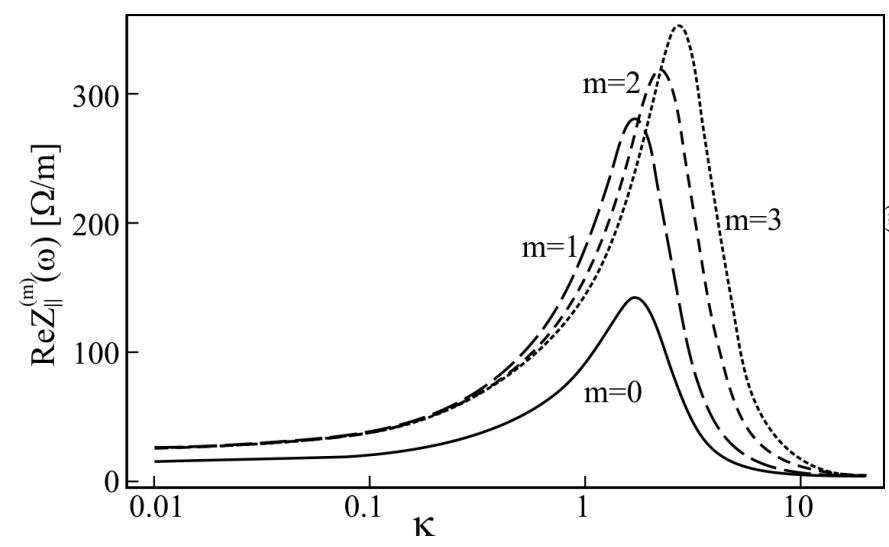

$$
U_{1}=\frac{a_{2}^{2}}{2}+\frac{\varepsilon_{0}+\varepsilon}{\varepsilon_{0} \chi^{2}}\left[1-a_{2} \chi \frac{R_{3}^{\prime}\left(a_{2}\right)}{R_{3}\left(a_{2}\right)}\right] .
$$

When the skin depth is small in comparison with the radius, we obtain

$$
U_{1}=\frac{a^{2}}{2}+\frac{1}{\chi^{2}} \frac{\varepsilon_{03}}{\varepsilon_{0}}+\frac{\varepsilon_{03} a}{\varepsilon_{0} \chi} c t h \chi d .
$$

For the single-layer tube with infinity wall thickness $\left(a_{3} \rightarrow\right.$ $\infty$ ) the multipole impedance (36) is modified to

$$
U_{m}=\frac{a_{2}^{2}}{m+1}+\frac{\varepsilon_{0}+\varepsilon}{\varepsilon_{0} \chi^{2}}\left(m-\frac{a_{2} \chi K_{m}^{\prime}\left(a_{2} \chi\right)}{K_{m}\left(a_{2} \chi\right)}\right)
$$

which for $\left|a_{2} \chi\right| \gg m$ turns to the well-known truncated longitudinal multipole mode presentation [1]

$$
U_{m}=\frac{a_{2}^{2}}{m+1}+\frac{a_{2}}{\chi \varepsilon_{0}}\left(\varepsilon_{0}+\varepsilon\right) .
$$

\section{NUMERICAL EXAMPLES}

In this section we present numerical examples for the frequency dependent part of the longitudinal impedance $Z_{z m}(k)$.

In Fig. 2 the multipole longitudinal impedances for $m=$ $0,1,2,3$ are presented for the stainless steel (SS) tube with thin inner copper layer of thickness $\Delta=100 \mathrm{~nm}$. The radius and thickness of the chamber are equal to $2 \mathrm{~mm}$. The horizontal axis is scaled by the dimensionless wave number $\kappa=k s_{0}$ for the copper tube, where $s_{0}=$ $\left(2 c a_{2}^{2} \varepsilon_{0} / \sigma_{c}\right)^{1 / 3}$ is a characteristic distance of the tube and $\sigma_{c}$ is the copper conductivity.

The transition of the longitudinal dipole mode impedance from the copper tube to the stainless steel vacuum chamber is presented in Fig. 3. Shown are the impedances of stainless steel-copper two-layer vacuum chamber for

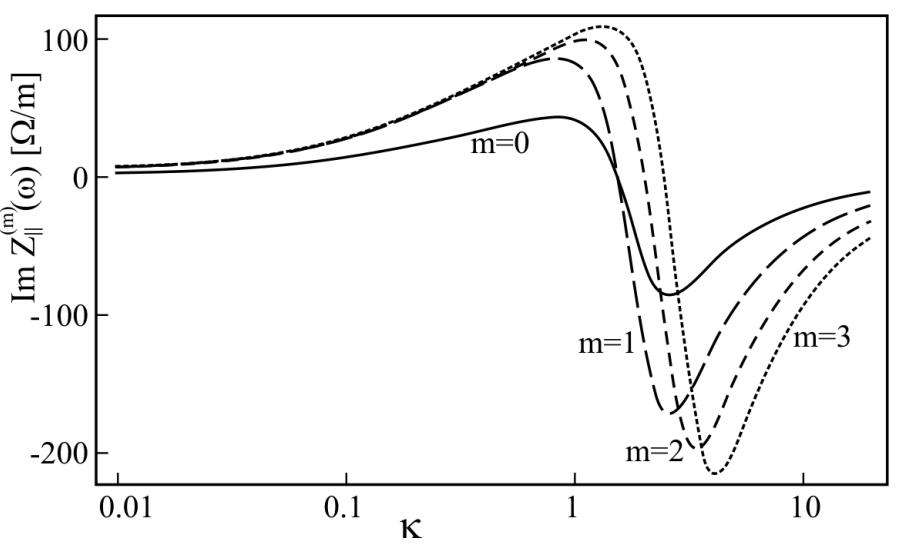

FIG. 2. The real (left) and imaginary (right) parts of longitudinal modes $(m=0,1,2,3)$ for stainless steel-copper tube. Copper layer thickness $\Delta=100 \mathrm{~nm}$. 


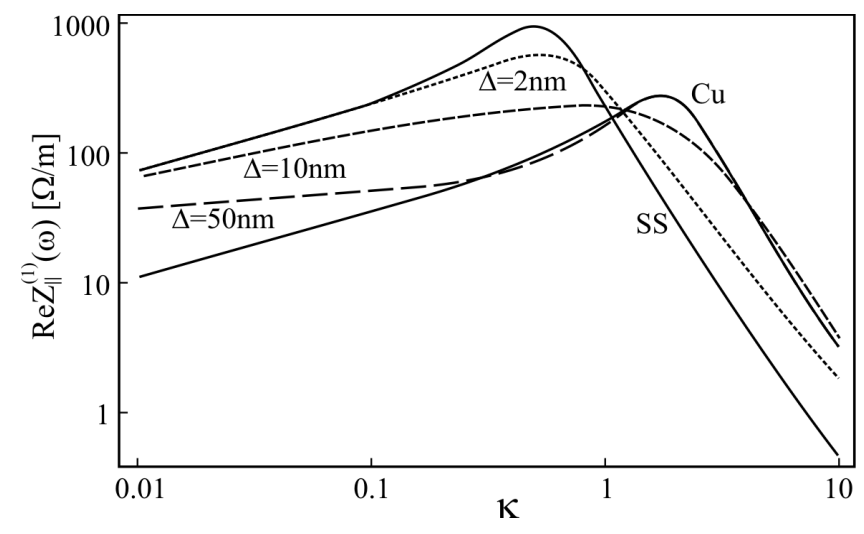

FIG. 3. The real part of stainless steel-copper tube longitudinal dipole impedance for various thickness of the inner copper layer. Tube inner radius is $2 \mathrm{~mm}$. The smooth curves present the ordinary copper $(\mathrm{Cu})$ and stainless steel (SS) single-layer tube impedances. $50 \mathrm{~nm}, 10 \mathrm{~nm}, 2 \mathrm{~nm}$ ).

$$
\begin{aligned}
S_{2}^{\prime}\left(a_{1}\right) R_{2}\left(a_{1}\right)-S_{2}\left(a_{1}\right) R_{2}^{\prime}\left(a_{1}\right) & =\left(a_{1} a_{2} \lambda^{2}\right)^{-1}, \\
S_{4}^{\prime}\left(a_{3}\right) R_{4}\left(a_{3}\right)-S_{4}\left(a_{3}\right) R_{4}^{\prime}\left(a_{3}\right) & =\left(a_{3} a_{4} \chi_{4}^{2}\right)^{-1}, \\
R_{2}^{\prime}\left(a_{1}\right) I_{m}\left(\lambda a_{1}\right)-R_{2}\left(a_{1}\right) I_{m}^{\prime}\left(\lambda a_{1}\right) & =I_{m}\left(\lambda a_{2}\right) / \lambda a_{1},
\end{aligned}
$$

various thickness of the copper layer $(\Delta=$

\section{CONCLUSION}

The exact solution for the longitudinal and transverse impedance multipoles in two-layer vacuum chamber with circular cross section is obtained. Several special cases are evaluated that coincide with well-known results.

The results of the work can be effectively applied for exact evaluation of the longitudinal and transverse impedances of the laminated vacuum chamber in modern accelerators.

\section{ACKNOWLEDGMENTS}

The authors express thanks to Vasili Tsakanov for many stimulating discussions and very useful comments.

\section{APPENDIX}

The matching of the field components at the subsequent boundaries $r=a_{1}, a_{2}, a_{3}, a_{4}$ (27) leads to system of 16 algebraic equations with 16 unknown coefficients $F_{i}, P_{i}$ $(i=1, \ldots, 5)$, and $Q_{i}, G_{i}(i=2,3,4)$. To modify the system of equations the following relations will be in used:

$$
\begin{aligned}
& S_{3}^{\prime}\left(a_{2}\right) R_{3}\left(a_{2}\right)-S_{3}\left(a_{2}\right) R_{3}^{\prime}\left(a_{2}\right)=\left(a_{2} a_{3} \chi_{3}^{2}\right)^{-1}, \\
& S_{2}^{\prime}\left(a_{1}\right) I_{m}\left(\lambda a_{1}\right)-S_{2}\left(a_{1}\right) I_{m}^{\prime}\left(\lambda a_{1}\right)=I_{m}^{\prime}\left(\lambda a_{2}\right) / \lambda a_{1},
\end{aligned}
$$

and

$$
R_{i}\left(a_{i}\right)=0, \quad S_{i}\left(a_{i}\right)=-\left(\chi_{i} a_{i}\right)^{-1}, \quad S_{i}^{\prime}\left(a_{i}\right)=0, \quad R_{i}^{\prime}\left(a_{i}\right)=\left(\chi_{i} a_{i}\right)^{-1} \quad(i=2,3,4) .
$$

Denoting $G_{1}=G_{1}\left(a_{1}\right)$, for the first boundary $(i=1)$ we get

$$
\left\{\begin{array}{l}
F_{1} \cdot I_{m}\left(\lambda a_{1}\right)+G_{1}=F_{2} \cdot R_{2}\left(a_{1}\right)+G_{2} \cdot S_{2}\left(a_{1}\right), \\
F_{1} \cdot I_{m}^{\prime}\left(\lambda a_{1}\right)+\frac{m}{\lambda a_{1}} G_{1}=F_{2} \cdot R_{2}^{\prime}\left(a_{1}\right)+G_{2} \cdot S_{2}^{\prime}\left(a_{1}\right), \\
P_{1} \cdot I_{m}\left(\lambda a_{1}\right)=P_{2} \cdot R_{2}\left(a_{1}\right)+Q_{2} \cdot S_{2}\left(a_{1}\right), \\
P_{1} \cdot I_{m}^{\prime}\left(\lambda a_{1}\right)=P_{2} \cdot R_{2}^{\prime}\left(a_{1}\right)+Q_{2} \cdot S_{2}^{\prime}\left(a_{1}\right) .
\end{array}\right.
$$

The linear combination of these equations gives

$$
i=1\left\{\begin{array}{l}
-G_{2}=F_{1} \lambda a_{2} I_{m}\left(\lambda a_{2}\right)+G_{1} \cdot\left[\lambda a_{1} R_{2}^{\prime}\left(a_{1}\right)-m R_{2}\left(a_{1}\right)\right] \lambda a_{2}, \\
F_{2}=F_{1} \lambda a_{2} I_{m}^{\prime}\left(\lambda a_{2}\right)+G_{1} \cdot \lambda a_{2}\left[\lambda a_{1} S_{2}^{\prime}\left(a_{1}\right)-m S_{2}\left(a_{1}\right)\right], \\
-L_{2}=P_{1} \lambda a_{2} I_{m}\left(\lambda a_{2}\right), \\
P_{2}=P_{1} \lambda a_{2} I_{m}^{\prime}\left(\lambda a_{2}\right) .
\end{array}\right.
$$

Performing the similar modifications for the matching equations at the subsequent boundaries $i=2,3,4$ and taking into account the relations (A1) and (A2), we obtain $i=2$

$$
i=2\left\{\begin{array}{l}
G_{3}=\frac{a_{3} \chi_{3}^{2}}{\lambda}\left[F_{2} \cdot \alpha_{1} R_{3}\left(a_{2}\right)+G_{2} \cdot R_{3}^{\prime}\left(a_{2}\right)-L_{2} \cdot m \alpha_{2} R_{3}\left(a_{2}\right)\right], \\
-F_{3}=\frac{a_{3} \chi_{3}^{2}}{\lambda}\left[F_{2} \cdot \alpha_{1} S_{3}\left(a_{2}\right)+G_{2} \cdot S_{3}^{\prime}\left(a_{2}\right)-L_{2} \cdot m \alpha_{2} S_{3}\left(a_{2}\right)\right], \\
L_{3}=\frac{a_{3} \chi_{3}^{2}}{\lambda}\left[P_{2} \cdot \alpha_{3} R_{3}\left(a_{2}\right)+L_{2} \cdot R_{3}^{\prime}\left(a_{2}\right)-G_{2} \cdot m \alpha_{4} R_{3}\left(a_{2}\right)\right], \\
-P_{3}=\frac{a_{3} \chi_{3}^{2}}{\lambda}\left[P_{2} \cdot \alpha_{3} S_{3}\left(a_{2}\right)+L_{2} \cdot S_{3}^{\prime}\left(a_{2}\right)-G_{2} \cdot m \alpha_{4} S_{3}\left(a_{2}\right)\right] ;
\end{array}\right.
$$




$$
\begin{gathered}
i=3\left\{\begin{array}{l}
G_{4}=\frac{a_{4} \chi_{4}^{2}}{\chi_{3}}\left[F_{3} \cdot \alpha_{5} R_{4}\left(a_{3}\right)+G_{3} \cdot R_{4}^{\prime}\left(a_{3}\right)-L_{3} \cdot m \alpha_{6} R_{4}\left(a_{3}\right)\right] \\
-F_{4}=\frac{a_{4} \chi_{4}^{2}}{\chi_{3}}\left[F_{3} \cdot \alpha_{5} S_{4}\left(a_{3}\right)+G_{3} \cdot S_{4}^{\prime}\left(a_{3}\right)-L_{3} \cdot m \alpha_{6} S_{4}\left(a_{3}\right)\right], \\
L_{4}=\frac{a_{4} \chi_{4}^{2}}{\chi_{3}}\left[P_{3} \cdot \alpha_{7} R_{4}\left(a_{3}\right)+L_{3} \cdot R_{4}^{\prime}\left(a_{3}\right)-G_{3} \cdot m \alpha_{8} R_{4}\left(a_{3}\right)\right], \\
-P_{4}=\frac{a_{4} \chi_{4}^{2}}{\chi_{3}}\left[P_{3} \cdot \alpha_{7} S_{4}\left(a_{3}\right)+L_{3} \cdot S_{4}^{\prime}\left(a_{3}\right)-G_{3} \cdot m \alpha_{8} S_{4}\left(a_{3}\right)\right]
\end{array}\right. \\
i=4\left\{\begin{array}{c}
F_{4} \cdot \alpha_{9}-L_{4} \cdot m \alpha_{10}=-G_{4} \cdot \frac{K_{m}^{\prime}\left(\lambda a_{4}\right)}{K_{m}\left(\lambda a_{4}\right)} \\
P_{4} \cdot \alpha_{11}-G_{4} \cdot m \alpha_{12}=-L_{4} \cdot \frac{K_{m}^{\prime}\left(\lambda a_{4}\right)}{K_{m}\left(\lambda a_{4}\right)}
\end{array}\right.
\end{gathered}
$$

where we use next notations

$$
\begin{aligned}
\alpha_{1} & =\frac{\chi_{3} \varepsilon_{0}}{\lambda \varepsilon_{3}}, \quad \alpha_{2}=\frac{1}{v a_{2} \lambda^{2} \chi_{3} \varepsilon_{3}}\left[\chi_{3}^{2}-\lambda^{2}\right], \quad \alpha_{3}=\frac{\chi_{3}}{\lambda}, \quad \alpha_{4}=\frac{v}{a_{2} \lambda^{2} \chi_{3}}\left[\varepsilon_{0} \chi_{3}^{2}-\varepsilon_{3} \lambda^{2}\right], \quad \alpha_{5}=\frac{\chi_{4} \varepsilon_{3}}{\chi_{3} \varepsilon_{4}}, \\
\alpha_{6} & =\frac{1}{v a_{3} \chi_{3}^{2} \chi_{4} \varepsilon_{4}}\left[\chi_{4}^{2}-\chi_{3}^{2}\right], \quad \alpha_{7}=\frac{\chi_{4}}{\chi_{3}}, \quad \alpha_{8}=\frac{v}{a_{3} \chi_{3}^{2} \chi_{4}}\left[\varepsilon_{3} \chi_{4}^{2}-\varepsilon_{4} \chi_{3}^{2}\right], \quad \alpha_{9}=\frac{\lambda \varepsilon_{4}}{\chi_{4} \varepsilon_{0}}, \\
\alpha_{10} & =\frac{1}{v a_{4} \chi_{4}^{2} \lambda \varepsilon_{0}}\left[\lambda^{2}-\chi_{4}^{2}\right], \quad \alpha_{11}=\frac{\lambda}{\chi_{4}}, \quad \alpha_{12}=\frac{v}{a_{4} \chi_{4}^{2} \lambda}\left[\varepsilon_{4} \lambda^{2}-\varepsilon_{0} \chi_{4}^{2}\right] .
\end{aligned}
$$

Substituting (A3) into (A4) we get

$$
\left\{\begin{array}{l}
F_{3}=I_{m}\left(\lambda a_{2}\right) \frac{a_{2} a_{3} \chi_{3}^{2}}{\lambda^{2}}\left[F_{1} \cdot \beta_{1}+P_{1} \cdot m \beta_{2}+G_{1} \cdot \beta_{3}\right] \\
G_{3}=I_{m}\left(\lambda a_{2}\right) \frac{a_{2} a_{3} \chi_{3}^{2}}{\lambda^{2}}\left[F_{1} \cdot \beta_{4}+P_{1} \cdot m \beta_{5}+G_{1} \cdot \beta_{6}\right] \\
P_{3}=I_{m}\left(\lambda a_{2}\right) \frac{a_{2} a_{3} \chi_{3}^{2}}{\lambda^{2}}\left[F_{1} \cdot m \beta_{7}+P_{1} \cdot \beta_{8}+G_{1} \cdot m \beta_{9}\right] \\
L_{3}=I_{m}\left(\lambda a_{2}\right) \frac{a_{2} a_{3} \chi_{3}^{2}}{\lambda^{2}}\left[F_{1} \cdot m \beta_{10}+P_{1} \cdot \beta_{11}+G_{1} \cdot m \beta_{12}\right]
\end{array}\right.
$$

where

$$
\begin{array}{ll}
\beta_{1}=S_{3}^{\prime}\left(a_{2}\right) \lambda^{2}-\frac{\beta \alpha_{1}}{\lambda a_{2}} S_{3}\left(a_{2}\right), & \beta_{2}=-S_{3}\left(a_{2}\right) \alpha_{2}, \quad \beta_{3}=S_{3}^{\prime}\left(a_{2}\right) \lambda^{2} W_{1}-\frac{\alpha_{1}}{\lambda a_{2}} S_{3}\left(a_{2}\right) W_{2}, \\
\beta_{4}=\frac{\beta \alpha_{1}}{\lambda a_{2}} R_{3}\left(a_{2}\right)-R_{3}^{\prime}\left(a_{2}\right) \lambda^{2}, & \beta_{5}=R_{3}\left(a_{2}\right) \alpha_{2}, \quad \beta_{6}=\frac{\alpha_{1}}{\lambda a_{2}} R_{3}\left(a_{2}\right) W_{2}-R_{3}^{\prime}\left(a_{2}\right) \lambda^{2} W_{1}, \quad \beta_{7}=-S_{3}\left(a_{2}\right) \alpha_{6}, \\
\beta_{8}=S_{3}^{\prime}\left(a_{2}\right) \lambda^{2}-\frac{\beta \alpha_{5}}{\lambda a_{2}} S_{3}\left(a_{2}\right), & \beta_{9}=-S_{3}\left(a_{2}\right) \alpha_{6} W_{1}, \quad \beta_{10}=R_{3}\left(a_{2}\right) \alpha_{6}, \\
\beta_{11}=\frac{\beta \alpha_{5}}{\lambda a_{2}} R_{3}\left(a_{2}\right)-R_{3}^{\prime}\left(a_{2}\right) \lambda^{2}, & \beta_{12}=R_{3}\left(a_{2}\right) \alpha_{6} W_{1},
\end{array}
$$

with $W_{1}=\lambda a_{1} R_{2}^{\prime}\left(a_{1}\right)-m R_{2}\left(a_{1}\right), W_{2}=\lambda a_{2}\left[\lambda a_{1} S_{2}^{\prime}\left(a_{1}\right)-m S_{2}\left(a_{1}\right)\right], \beta=\left[\lambda a_{2} I_{m}^{\prime}\left(\lambda a_{2}\right)\right] / I_{m}\left(\lambda a_{2}\right)$.

Substituting (A8) into (A5) we get

$$
\left\{\begin{array}{l}
F_{4}=I_{n}\left(\lambda a_{2}\right) \frac{a_{2} a_{3} a_{4} \chi_{4}^{2}}{\lambda^{2}}\left[F_{1} \cdot \theta_{1}+P_{1} \cdot m \theta_{2}+G_{1} \cdot \theta_{3}\right], \\
-G_{4}=I_{n}\left(\lambda a_{2}\right) \frac{a_{2} a_{3} a_{4} \chi_{4}^{2}}{\lambda^{2}}\left[F_{1} \cdot \theta_{4}+P_{1} \cdot m \theta_{5}+G_{1} \cdot \theta_{6}\right] \\
P_{4}=I_{n}\left(\lambda a_{2}\right) \frac{a_{2} a_{3} a_{4} \chi_{4}^{2}}{\lambda^{2}}\left[F_{1} \cdot m \theta_{7}+P_{1} \cdot \theta_{8}+G_{1} \cdot m \theta_{9}\right], \\
-L_{4}=I_{n}\left(\lambda a_{2}\right) \frac{a_{2} a_{3} a_{4} \chi_{4}^{2}}{\lambda^{2}}\left[F_{1} \cdot m \theta_{10}+P_{1} \cdot \theta_{11}+G_{1} \cdot m \theta_{12}\right]
\end{array}\right.
$$

where 


$$
\begin{aligned}
& \theta_{1}=S_{4}\left(a_{3}\right)\left[m^{2} \alpha_{4} \beta_{10}-\alpha_{3} \beta_{1}\right]-S_{4}^{\prime}\left(a_{3}\right) \beta_{4} \chi_{3}^{2}, \quad \theta_{2}=S_{4}\left(a_{3}\right)\left[\alpha_{4} \beta_{11}-\alpha_{3} \beta_{2}\right]-S_{4}^{\prime}\left(a_{3}\right) \beta_{5} \chi_{3}^{2}, \\
& \theta_{3}=S_{4}\left(a_{3}\right)\left[m^{2} \alpha_{4} \beta_{12}-\alpha_{3} \beta_{3}\right]-S_{4}^{\prime}\left(a_{3}\right) \beta_{6} \chi_{3}^{2}, \quad \theta_{4}=R_{4}\left(a_{3}\right)\left[m^{2} \alpha_{4} \beta_{10}-\alpha_{3} \beta_{1}\right]-R_{4}^{\prime}\left(a_{3}\right) \beta_{4} \chi_{3}^{2} \text {, } \\
& \theta_{5}=R_{4}\left(a_{3}\right)\left[\alpha_{4} \beta_{11}-\alpha_{3} \beta_{2}\right]-R_{4}^{\prime}\left(a_{3}\right) \beta_{5} \chi_{3}^{2}, \quad \theta_{6}=R_{4}\left(a_{3}\right)\left[m^{2} \alpha_{4} \beta_{12}-\alpha_{3} \beta_{3}\right]-R_{4}^{\prime}\left(a_{3}\right) \beta_{6} \chi_{3}^{2}, \\
& \theta_{7}=S_{4}\left(a_{3}\right)\left[\alpha_{8} \beta_{4}-\alpha_{7} \beta_{7}\right]-S_{4}^{\prime}\left(a_{3}\right) \beta_{10} \chi_{3}^{2}, \quad \theta_{8}=S_{4}\left(a_{3}\right)\left[m^{2} \alpha_{8} \beta_{5}-\alpha_{7} \beta_{8}\right]-S_{4}^{\prime}\left(a_{3}\right) \beta_{11} \chi_{3}^{2}, \\
& \theta_{9}=S_{4}\left(a_{3}\right)\left[\alpha_{8} \beta_{6}-\alpha_{7} \beta_{9}\right]-S_{4}^{\prime}\left(a_{3}\right) \beta_{12} \chi_{3}^{2}, \quad \theta_{10}=R_{4}\left(a_{3}\right)\left[\alpha_{8} \beta_{4}-\alpha_{7} \beta_{7}\right]-R_{4}^{\prime}\left(a_{3}\right) \beta_{10} \chi_{3}^{2}, \\
& \theta_{11}=R_{4}\left(a_{3}\right)\left[m^{2} \alpha_{8} \beta_{5}-\alpha_{7} \beta_{8}\right]-R_{4}^{\prime}\left(a_{3}\right) \beta_{11} \chi_{3}^{2}, \quad \theta_{12}=R_{4}\left(a_{3}\right)\left[\alpha_{8} \beta_{6}-\alpha_{7} \beta_{9}\right]-R_{4}^{\prime}\left(a_{3}\right) \beta_{12} \chi_{3}^{2}
\end{aligned}
$$

Substituting (A9) into (A6) we get

and the function $A_{m}(\lambda, k)$ in ultrarelativistic limit is given by

$$
A_{m}(\lambda, k) \underset{\lambda \rightarrow 0}{\rightarrow}\left(\frac{a_{2}}{a_{1}}\right)^{m}\left[1+\frac{a_{1}^{2}}{(m+1) U_{m}(k)}\left(\frac{a_{1}}{a_{2}}\right)^{2 m}\right],
$$

where

$$
\begin{aligned}
& \tau_{1}=\alpha_{11} \theta_{1}-\frac{\alpha}{a_{4}} \theta_{4}+m^{2} \alpha_{12} \theta_{10}, \\
& \tau_{2}=\alpha_{11} \theta_{2}-\frac{\alpha}{a_{4}} \theta_{5}+\alpha_{12} \theta_{11}, \\
& \tau_{3}=\alpha_{11} \theta_{3}-\frac{\alpha}{a_{4}} \theta_{6}+m^{2} \alpha_{12} \theta_{12}, \\
& \tau_{4}=\alpha_{9} \theta_{7}-\frac{\alpha}{a_{4}} \theta_{10}+\alpha_{10} \theta_{4}, \\
& \tau_{5}=\alpha_{9} \theta_{8}-\frac{\alpha}{a_{4}} \theta_{11}+m^{2} \alpha_{10} \theta_{5}, \\
& \tau_{6}=\alpha_{9} \theta_{9}-\frac{\alpha}{a_{4}} \theta_{12}+\alpha_{10} \theta_{6},
\end{aligned}
$$

with $\alpha=\left[\lambda a_{4} K_{m}^{\prime}\left(\lambda a_{4}\right)\right] / K_{m}\left(\lambda a_{4}\right)$.

Finally by solving (A10) for coefficient $F_{1}$ we get

$$
\begin{aligned}
F_{1} & =-G_{1}\left(a_{1}\right) \frac{\tau_{3} \tau_{5}-m^{2} \tau_{2} \tau_{6}}{\tau_{1} \tau_{5}-m^{2} \tau_{2} \tau_{4}} \frac{1}{I_{m}\left(\lambda a_{2}\right)} \\
& =-\frac{G_{1}\left(a_{1}\right)}{I_{m}\left(\lambda a_{2}\right)} A_{m}(\lambda, k) .
\end{aligned}
$$

In ultrarelativistic limit $(\lambda \rightarrow 0)$, the following relations are valid:

$$
\begin{aligned}
\beta & =m, \quad \alpha=-m, \quad W_{1}=\left(a_{2} / a_{1}\right)^{m}, \\
W_{2} & =m \cdot\left(a_{2} / a_{1}\right)^{m},
\end{aligned}
$$

with the function $U_{m}(k)$ given explicitly in the main body of this paper.

[1] A. W. Chao, Physics of Collective Beam Instabilities in High Energy Accelerators (Wiley, New York, 1993).

[2] B. W.Zotter and S. A. Kheifetz, Impedances and Wakes in High-Energy Particle Accelerators (World Scientific, Singapore, 1997).

[3] H. Henke and O. Napoly, in Proceedings of the Second European Particle Accelerator Conference, Nice, France, 1990 (Editions Frontiers, Gif-sur-Yvette, 1991), pp. 1046-1048.

[4] A.W. Chao, Technical Report No. 2946, SLAC-PUB, 1982.

[5] A. Piwinski, Report No. DESY-94-068, 1994, p. 23.

[6] A. Burov and A. Novokhatskii, INP-Novosibirsk, Report No. 90-28, 1990.

[7] A. Burov and V. Lebedev, in Proceedings of the European Particle Accelerator Conference, Paris, France 2002 (EPS-IGA and CERN, Geneva, 2002), pp. 1452-1454.

[8] M. Ivanyan and V. Tsakanov, Phys. Rev. ST Accel. Beams 7, 114402 (2004).

[9] A. M. Al-khateeb, O. Boine-Frankenheim, R. W. Hasse and I. Hofmann, Phys. Rev. E 71, 026501 (2005).

[10] B. Zotter, Part. Accel. 1, 311 (1970).

[11] B. Zotter, CERN Technical Report No. 69-15, 1969.

[12] W. Panofsky and W. Wenzel, Rev. Sci. Instrum. 27, 967 (1956). 